\title{
Spatial Geometric Constraint Solving Based on k-connected Graph Decomposition*
}

\author{
Gui-Fang Zhang \\ School of Sciences \\ Beijing Forestry University \\ Beijing 100083, China
}

\author{
Xiao-Shan Gao \\ KLMM, Institute of Systems Science \\ Academia Sinica \\ Beijing 100080, China
}

\begin{abstract}
We propose a geometric constraint solving method based on connectivity analysis in graph theory, which can be used to decompose a well-constrained problem into some smaller ones if possible. We also show how to merge two rigid bodies if they share two or three geometric primitives in a biconnected or tri-connected graph respectively. Based on this analysis, problems similar to the "double banana problem" could be easily detected.
\end{abstract}

\section{Categories and Subject Descriptors J.6 [COMPUTER-AIDED ENGINEERING]: CAD}

\section{Keywords}

Geometric constraint solving, parametric CAD, k-connected graph, separating k-tuple, decomposition

\section{INTRODUCTION}

Geometric constraint solving(GCS) is one of the key techniques in parametric $\mathrm{CAD}$, which allows the user to make modifications to existing designs by changing parametric values. There are four major approaches to geometric constraint solving: the numerical approach, the symbolic computation approach, the rule-based approach, and the graphbased approach. This paper will focus on using graph algorithms to decompose a large constraint problem into smaller ones.

In [11], Owen proposed a GCS method based on the triconnected decomposition of graphs. In [1], Hoffmann et al proposed a method based on cluster formation to solve $2 \mathrm{D}$ and $3 \mathrm{D}$ constraint problems. In [5], Joan-Arinyo et al proposed an algorithm to decompose a $2 \mathrm{D}$ constraint problem into an s-tree. This method is equivalent to Owen's and Hoffmann's methods, but is conceptually simpler.

${ }^{*}$ Partially supported by a 973 project of China

Permission to make digital or hard copies of all or part of this work for personal or classroom use is granted without fee provided that copies are not made or distributed for profit or commercial advantage and that copies bear this notice and the full citation on the first page. To copy otherwise, to republish, to post on servers or to redistribute to lists, requires prior specific permission and/or a fee.

SAC'O6 April 23-27, 2006, Dijon, France

Copyright 2006 ACM 1-59593-108-2/06/0004 ...\$5.00.
The above approaches use triangles as basic patterns to solve geometric constraint problems. In [9], Latham and Middleditch proposed an algorithm which could be used to decompose a constraint problem into what we called general construction sequence[2]. A similar method based on maximal matching of bipartite graphs was proposed in [8]. In [4], Hoffmann et al gave an algorithm to find rigid bodies in a constraint problem. From this, several general approaches to GCS are proposed [4]. In [2], a c-tree decomposition method is proposed to solve both $2 \mathrm{D}$ and $3 \mathrm{D}$ constraint problems. The method proposed in [4] and the c-tree method [2] can be used to find a decomposition with the smallest controlling problem in certain sense. In [13], a method based on connectivity analysis from graph theory is proposed for solving $2 \mathrm{D}$ geometric constraint problems. This method is a natural generalization of the methods in $[5,11]$ which are based on tri-connectivity analysis of the constrained graph, and can solve problems that can be reduced to triangular form.

In this paper, we extend the results in [13] to 3D case. The method works as follows, we first decompose a wellconstrained constraint graph into $k$-connected subgraphs for the smallest possible $k$. If one of the subgraphs is also wellconstrained, then we can solve the original problem by first solving the subgraphs and then merging these subgraphs. The merging process is carefully analyzed. As a result, the "double banana problem" could be easily detected in the algorithm.

In Section 2, we introduce the concept of connected graph. In Section 3, the method to split constraint graph is proposed. In Section 4, an algorithm to generate the D-tree is proposed. In Section 5, a method to merge bi-connected and tri-connected constraint graphs is proposed.

\section{PRELIMINARY RESULTS}

The geometric primitives considered are points, lines and planes in 3D. The geometric constraints considered include distance constraints between point/point, point/line, point /plane, line/line and the angular constraints between line/line, line/plane, plane/plane in 3D.

We use a constraint graph to represent a constraint problem. The vertices of the graph represent the geometric primitives and the edges represent the constraints. Let $G=$ $(V, E, \omega)$ (or $G=(V(G), E(G), \omega))$ be a geometric constraint graph. $\forall v \in V, \omega(v)$ is the weight of vertex $v$, i.e the number of independent parameters used to determine the vertex, and $\omega(V)=\Sigma_{v \in V} \omega(v)$. For instance, the weight of every point or plane is 3 in $3 \mathrm{D}$ and the weight of a line in $3 \mathrm{D}$ is 4 . $\forall e \in E, \omega(e)$ represents the weight of edge $e$, i.e 
the number of scalar equations to represent the constraint, and $\omega(E)=\Sigma_{e \in E} \omega(e)$. For instance, the weight of the distance constraint between two points is 1 if the distance is not zero, otherwise it is 3 . Let $G=(V, E, \omega)$ be a geometric constraint graph.

1. $G$ is structurally over-constrained if there is an induced subgraph $H=\left(V_{H}, E_{H}\right)$ satisfying $\omega\left(E_{H}\right)>\omega\left(V_{H}\right)-$ 6 , where $\left|V_{H}\right|>2$.

2. G is structurally under-constrained if it is not structurally over-constrained and $0<\omega(E)<\omega(V)-6$.

3. $G$ is structurally well-constrained if it is neither structurally under-constrained nor over-constrained.

Let $G=(V, E, \omega)$ be a geometric constraint graph in 3D. We define the deficit function associated with $G$ as

$$
\operatorname{deficit}(G)=(\omega(V)-6)-\omega(E) .
$$

If $G$ is a structurally well-constrained problem, $\operatorname{deficit}(G)=$ 0 . If $\mathrm{G}$ is not structurally over-constrained, $\operatorname{deficit}(G) \geq 0$.

An undirected graph $G=(V, E)$ is called connected if for every two nodes $x, y \in V$ there exists a path of edges from $\mathrm{E}$ joining $\mathrm{x}$ and $\mathrm{y}$. A graph is called disconnected if it is not connected. A graph is called $k$-connected $(k \geq 1)$ if there does not exit a set of $k-1$ or fewer nodes $V^{\prime} \subseteq V$ such that the removal of all nodes of $V^{\prime}$ and their incident edges from $G$ results in a disconnected graph.

Two vertices $\mathrm{x}$ and $\mathrm{y}$ of graph $\mathrm{G}$ are said to be k-connected if $\mathrm{k}$ is the largest integer such that there exist $\mathrm{k}$ vertexdisjoint paths from $\mathrm{x}$ to $\mathrm{y}$ in $\mathrm{G}$. The connectivity of $\mathrm{x}$ and $\mathrm{y}$ is denoted by $\kappa(x, y)$, which is the maximal number of vertex disjoint paths from $\mathrm{x}$ to $\mathrm{y}$ in $\mathrm{G}$.

Theorem 2.1 ([6]). (Theorem of Whitney) A graph $G$ is $k$-connected if and only if $\kappa(x, y) \geq k$ for any two vertices $x$ and $y$ of $G$, that is, $\kappa(G)=\min \{\kappa(x, y): x, y \in V\}$.

The complexity of the algorithm to calculate the connectivity of a connected graph $G=(V, E)$ is $O\left(|V|^{\frac{1}{2}}|E|^{2}\right)[6]$.

THEOREM 2.2. Let $G$ be a well-constrained graph. We have

$$
\kappa(G) \leq \quad \begin{aligned}
& 5 \text { in } 3 D \text { for points and planes } \\
& 7 \text { in } 3 D \text { for points, planes and lines. }
\end{aligned}
$$

Proof. For a graph $G=(V, E)$, from [10] it is known that $\kappa(G) \leq \frac{2|E|}{|V|}$. Then for a structurally well-constrained constraint graph $G=(V, E)$, we can obtain the bound of $\kappa(G)$ explicitly as follows.

1. If the primitives are points and planes, then $|E| \leq$ $3|V|-6$ and $\frac{2|E|}{|V|} \leq \frac{2(3|V|-6)}{|V|}<6$. Thus $\kappa(G) \leq 5$.

2. If the geometric primitives are points, lines and planes, then let $V_{3}$ be the set of all vertices of weight three, $|E| \leq 3\left|V_{3}\right|+4\left(|V|-\left|V_{3}\right|\right)-6=4|V|-\left|V_{3}\right|-6$. Thus $\frac{2|E|}{|V|} \leq \frac{2\left(4|V|-\left|V_{3}\right|-6\right)}{|V|}<8$ and $\kappa(G) \leq 7$.

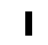

Let $G=(V, E, \omega)$ be a connected undirected graph. A vertex $v \in V$ is a separating vertex for $\mathrm{G}$ if the induced subgraph by $V-\{v\}$ is not connected. $G$ is bi-connected if it contains no separating vertex.
A pair of vertices $v_{1}, v_{2} \in V$ is a separating pair for $\mathrm{G}$ if the induced subgraph on $V-\left\{v_{1}, v_{2}\right\}$ is not connected. G is triconnected if it contains no separating pairs and vertices[3].

A triplet $\left\{v_{1}, v_{2}, v_{3}\right\}$ of distinct vertices in $\mathrm{V}$ is a separating triplet of a tri-connected graph if the subgraph induced by $V-\left\{v_{1}, v_{2}, v_{3}\right\}$ is not connected. $\mathrm{G}$ is 4 -connected if it contains no separating triplets, pairs and vertices [7].

A tuple $\left\{v_{1}, v_{2}, \cdots, v_{k}\right\}$ of distinct vertices in $\mathrm{V}$ is a separating $k$-tuple of a k-connected graph if the subgraph induced by $V-\left\{v_{1}, v_{2}, \cdots, v_{k}\right\}$ is not connected. $\mathrm{G}$ is $(k+1)$ connected if it contains no separating n-tuple $\left\{v_{1}, v_{2}, \cdots, v_{n}\right\}$ $(n=1, \cdots, k)$.

\section{SPLIT AND CUT GRAPHS}

In this section, let $G=(V, E, \omega)$ be a structurally wellconstrained geometric constraint graph. A subgraph $G_{s}$ in $G$ is called a cut graph of $G$ if $G$ is not connected after deleting $G_{s}$ and the edges adjacent to vertices in $G_{s}$. The ways to find cut graphs of $G$ are as follows.

1. Assuming that $G$ is $k$-connected and $\left\{v_{1}, v_{2}, \cdots, v_{k}\right\}$ is a separating k-tuple, where $v_{i} \in V(i=1, \cdots, k)$. The subgraph of $G$ induced by $\left\{v_{1}, v_{2}, \cdots, v_{k}\right\}$ is a cut graph.

2. Assuming that $H$ is a structurally well-constrained subgraph in $G$, i.e. $\operatorname{deficit}(H)=0$, let

$V_{c}=\left\{v \mid\left(v, v_{1}\right) \in E(G), v \in V(H), v_{1} \in(V(G)-V(H))\right\}$, if $|V(H)|>\left|V_{c}\right|$, the subgraph of $G$ induced by $V_{c}$ is a cut graph associated with $H$.

A $k$-connected graph can be split into split components by splitting it at the separating $k$-tuple, i.e. deleting the separating $k$-tuple and edges adjacent to the $k$-tuple.

Let $V_{s}=\left\{v_{1}, v_{2}, \cdots, v_{k}\right\}$ be the separating k-tuple in the graph $G$ that induces the split components $C^{1}, C^{2}, \cdots, C^{n}$.

If there exists a split component $C^{j}(0 \leq j \leq n)$ satisfying $\operatorname{deficit}\left(C^{j}\right)=0$ and we can find a cut graph $G_{s}$ associated with $C^{j}$. Let $G_{1}=C^{j}$ and $G_{2}$ the subgraph of $G$ induced by $\left(V(G)-V\left(C^{j}\right)\right) \cup V_{s} . G_{1}$ and $G_{2}$ are called the split graphs of $G$.

For each $C^{i}(i=1, \cdots, n)$, if we duplicate $V_{s}$ to each $C^{i}$, the subgraph of $G$ induced by $V_{s} \cup V\left(C^{i}\right)$ is called split component and denoted by $C_{i}$.

If there exists a split component $C_{k}(0 \leq k \leq n)$ satisfies $\operatorname{deficit}\left(C_{k}\right)=0$, we can find a cut graph $G_{s}$ associated with $C_{k}$. Let $G_{1}=C_{k}$ and $G_{2}=\bigcup_{i=1, i \neq k}^{n} C_{i} . G_{1}$ and $G_{2}$ are called the split graphs of $G$.

For each $C_{i}(i=1, \cdots, n)$, if we can not find a cut graph associated with $C_{i}$, select an $m$ such that $1 \leq m \leq n$ and let

$$
G_{1}=\bigcup_{i=1}^{m} C_{i} ; \quad G_{2}=\bigcup_{i=m+1}^{n} C_{i} .
$$

The graph $G_{1}$ and $G_{2}$ are called the split graphs of $G$.

The relation of split graphs and the cut graph is shown in Fig.1.

TheOREM 3.1. Let $G$ be a k-connected structurally wellconstrained graph, $G_{s}=\left(V\left(G_{s}\right), E\left(G_{s}\right), \omega\right)$ the cut graph, $G_{1}=\left(V\left(G_{1}\right), E\left(G_{1}\right), \omega\right)$ and $G_{2}=\left(V\left(G_{2}\right), E\left(G_{2}\right), \omega\right)$ the split graphs. We have

$$
\operatorname{deficit}\left(G_{1}\right)+\operatorname{deficit}\left(G_{2}\right)=\operatorname{deficit}\left(G_{s}\right) .
$$




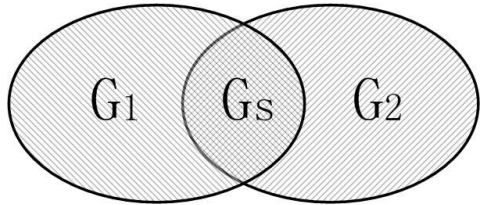

Figure 1: Relation between split graphs and cut graph

Proof: Since $G$ is structurally well-constrained, $\operatorname{deficit}\left(G_{s}\right) \geq$ 0 , $\operatorname{deficit}\left(G_{1}\right) \geq 0$ and $\operatorname{deficit}\left(G_{2}\right) \geq 0$. If $\operatorname{deficit}\left(G_{s}\right)>0$, $\operatorname{deficit}\left(G_{1}\right)>0$ and deficit $\left(G_{2}\right)>0$, then $\omega\left(V_{s}\right)-E_{s}-D>0$, $\omega\left(V\left(G_{1}\right)\right)-\omega\left(E\left(G_{1}\right)\right)-D>0$ and $\omega\left(V\left(G_{2}\right)\right)-\omega\left(E\left(G_{2}\right)\right)-$ $D>0$.

Because

$\omega\left(E\left(G_{1}\right)\right)+\omega\left(E\left(G_{2}\right)\right)-\omega\left(E_{s}\right)=\omega(V)-D$,

we have $\omega\left(E\left(G_{1}\right)\right)+\omega\left(E\left(G_{2}\right)\right)-\omega\left(E_{s}\right)$

$=\omega\left(V\left(G_{1}\right)\right)+\omega\left(V\left(G_{2}\right)\right)-\omega\left(V_{s}\right)-D$.

Then $\left(\omega\left(V\left(G_{1}\right)\right)-\omega\left(E\left(G_{1}\right)\right)-D\right)+\left(\omega\left(V\left(G_{2}\right)\right)\right.$

$\left.-\omega\left(E\left(G_{2}\right)\right)-D\right)=\omega\left(V_{s}\right)-\omega\left(E_{s}\right)-D$.

Thus $\operatorname{deficit}\left(G_{1}\right)+\operatorname{deficit}\left(G_{2}\right)=\operatorname{deficit}\left(G_{s}\right)$.

Corollary 3.2. Let $G=(V, E, \omega)$ be a structurally wellconstrained $k$-connected graph, $G_{s}=\left(V_{s}, E_{s}, \omega\right)$ the subgraph of $G$ induced by a separating $k$-tuple $V_{s}=\left\{v_{1}, v_{2}, \cdots\right.$, $\left.v_{k}\right\}, G_{1}$ and $G_{2}$ the split graphs of graph $G . G_{1}$ and $G_{2}$ are structurally well-constrained if and only if $G_{s}$ is structurally well-constrained.

Corollary 3.3. Let $G_{s}$ be the graph induced by a separating $k$-tuple of a $k$-connected structurally well-constrained graph $G, G_{1}$ and $G_{2}$ the split graphs. If $G_{s}$ is not structurally well-constrained and $G_{1}$ is structurally well-constrained, then $\operatorname{deficit}\left(G_{2}\right)=\operatorname{deficit}\left(G_{s}\right)$.

In general, a structurally well-constrained graph can be decomposed by the following ways based on connectivity analysis.

1. $\operatorname{deficit}\left(G_{s}\right)=0$. Now the split graphs $G_{1}$ and $G_{2}$ are structurally well-constrained by Corollary 3.2. We can solve them separately, then merge them to obtain the solutions to the initial problem. Fig. 2 is an example of this case. The graph in $3 \mathrm{D}$ is split into two structurally well-constrained graphs.
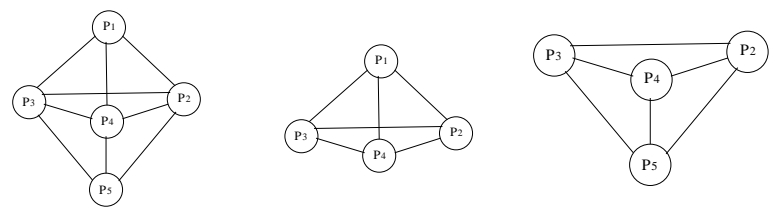

Figure 2: Separating triplet is $P_{2}, P_{3}, P_{4}$ and $\operatorname{deficit}\left(G_{s}\right)=0$

2. $\operatorname{deficit}\left(G_{s}\right)>0$. According to theorem 3.1, $\operatorname{deficit}\left(G_{s}\right)=$ $\operatorname{deficit}\left(G_{1}\right)+\operatorname{deficit}\left(G_{2}\right)$ and $\operatorname{deficit}\left(G_{1}\right)$ can be 0,1 , $\cdots$, deficit $\left(G_{s}\right)$. If either $G_{1}$ or $G_{2}$ is structurally wellconstrained, then let $G_{1}$ be the well-constrained split graph. We solve $G_{1}$ first, then add $\operatorname{deficit}\left(G_{s}\right)$ auxiliary constraints to $G_{s}$ so that $G_{s}$ becomes a structurally well-constrained problem $G_{s}^{\prime}$. Now, $G_{2}$ also becomes a well-constrained problem $G_{2}^{\prime}$, and can be solved separately. After getting the solution of $G_{1}$ and $G_{2}^{\prime}$, we can merge them to obtain a solution to the initial problem. $G_{2}^{\prime}$ is called the modified split graph of $G$ with $G_{1}$.

If neither $G_{1}$ nor $G_{2}$ is structurally well-constrained, we can make the following choices:

(a) Select another separating k-tuple and re-decompose the constraint graph $G$; or

(b) Solve $G$ with numerical computation.

In the cases $\operatorname{deficit}\left(G_{s}\right)>0$, we need to add auxiliary edges to make the cut graph $G_{s}$ well-constrained. This a well-constrained completion problem. Latham et al presented a method to solve such a problem [9].

\section{A DECOMPOSITION ALGORITHM}

We will introduce a new decomposition tree, D-tree, which can be used to simplify a structurally well-constrained constraint problem.

A $D$-tree for a structurally well-constrained graph $G=$ $(V, E)$ is a binary tree.

1. The root of the tree is the graph $G$. Left child $L$ and right child $R$ are defined as follows.

2. For each node $N$ in the tree, its left child $L$ is the split graph of $N$ which is either a triangle or a structurally well-constrained subgraph of $N$, and the right child $R$ is the (modified) split graph of $N$ with $L$ which is either a triangle or a structurally well-constrained graph.

3. All leaves are either a triangle or a structurally wellconstrained $j$-connected $(3 \leq j)$ constraint graph.

Algorithm 4.1. The input is a structurally well-constrained graph $G=(V(G), E(G), \omega)$ in $3 D$. The output is a $D$-tree for $G$. Let $T=G$ as the initial value, and $S_{k}=\emptyset$ as the initial value of set of separating $k$-tuples in $V(T)$.

S1 If $T$ is a triangle, the algorithm terminates; else goto step $\mathbf{S 2}$.

S2 Calculate connectivity $k$ of the connected graph T. If $|V(T)|-k<2$, the algorithm terminates; else let $S_{k} \leftarrow \emptyset$ goto $\mathbf{S 3}$.

S3 1. If $k=2$, find all the separating pairs with Hopcroft and Tarjon's method [3]. Then add these separating pairs to $S_{k}$, goto step $\mathbf{S} \mathbf{4}$.

2. If $k=3$, find all the separating triplets with the method in [7]. Then add these separating triplets to $S_{k}$, goto step $\mathbf{S} 4$.

3. If $k>3$, goto step $\mathbf{S 5}$.

S4 If $S_{k} \neq \varnothing$, taking a separating pair or triplet $S \in S_{k}$, $S_{k} \leftarrow S_{k}-\{S\}$, generate the cut graph $G_{s}$ induced by $S$, goto $\mathbf{S 7}$. Otherwise, the algorithm terminates.

S5 Let $H=T$. For all $k$-tuple $S=\left\{v_{1}, \cdots, v_{k}\right\}$ in $H$, do step S6. If we cannot find a separating t-tuple, the algorithm terminates. 
S6 Let $U$ be the graph induced by $H-S$. If $U$ is not connected, $S$ is a separating k-tuple. Generate the cut graph $G_{s}$ induced by $S$, goto $\mathbf{S 7}$. If $U$ is connected, goto step $S 5$.

S7 Splitting $T$ by the cut graph to generate the split graphs $G_{1}$ and $G_{2}$. If the deficit function of the cut graph induced by $S$ is 0 , goto $\mathbf{S 8}$; else goto $\mathbf{S 9}$.

S8 Let $L=G_{1}$ and $R=G_{2}$. Let $T=L$, repeat the algorithm from $\mathbf{S} 1$ recursively. Let $T=R$, repeat the algorithm from $\mathbf{S} 1$ recursively.

S9 If one of the split graph is structurally well-constrained, let it be $G_{1}$ and goto $\mathbf{S 1 0}$; else if neither $G_{1}$ nor $G_{2}$ is structurally well-constrained, goto S11.

$\mathbf{S 1 0}$ Let $L=T=G_{1}$ and operate the algorithm from $\mathbf{S 1}$ recursively for $T$. Add deficit $\left(G_{s}\right)$ auxiliary constraints to $G_{2}$ to make the modified split graph $G_{2}^{m}$ structurally well-constrained with the method proposed in [9]. Let $R$ be the modified split graph $G_{2}^{m}$, and $T=R$. Repeat the algorithm from $\mathbf{S} 1$ recursively.

$\mathbf{S 1 1}$ Check the separating component $C^{1}, C^{2}, \cdots, C^{n}$. If we can find a cut graph $G_{s}$ associated with $C^{j}(0 \leq j \leq n)$, goto $\mathbf{S 1 2}$; else if $k \leq 3$ goto $\mathbf{S 4}$; else if $k>3$ goto $\mathbf{S 5}$ to repeat the process for another $k$-tuple.

$\mathbf{S 1 2}$ Generate the split graphs $G_{1}$ and $G_{2}$. If $\operatorname{deficit}\left(G_{s}\right)=$ 0 , goto $\mathbf{S 8}$; else goto $\mathbf{S 1 0}$.

Let $n=|V|$. The complexity of step $\mathbf{S 2}$ is $n^{\frac{1}{2}}|E|^{2}[6]$. When $k=2$, the complexity of $\mathbf{S 3}$ is $O(n+|E|)$ [3]; when $k=3$, the complexity of $\mathbf{S 3}$ is $O\left(n^{2}\right)$ [7]. For Step S5, in the worst case, we need to consider $\frac{\mid n !}{k !(n-k) !} \mathrm{k}$-tuples. From Theorem 2.2, we know $k \leq 7$. Then the complexity is $O\left(n^{7}\right)$ at most to get all the separating $k$-tuples. So, the complexity to find the first split is $O\left(n^{7}\right)$. We need to repeat the algorithm for the two split graphs with $v_{1}$ and $v_{2}$ vertices $\left(v_{1}+v_{2}=n\right)$. Then the split process for the two split graphs are $O\left(v_{1}^{7}+v_{2}^{7}\right) \leq O\left(n^{7}\right)$. So the complexity of the algorithm is at most $O\left(n^{8}\right)$, and is hence polynomial in $n$.

\section{MERGE SPLIT GRAPHS}

After a D-tree is obtained for a geometric constraint problem, we can solve the problem as follows: Do a left to right depth first transversal of the D-tree and solve the constraint problem represented by each node as follows.

1. If the current node $N$ is a leaf in the tree then it is a well-constrained problem that cannot be decomposed further with the algorithm. Solve $N$ with numerical computation methods.

2. Let $N$ be a node with left child $L$ and right child $R$. This can be done in three steps.

(a) Solve the left child $L . L$ is a well-constrained problem which can be solved recursively.

(b) Solve the right child $R$. The values for the parameters in the auxiliary constraints in the right child $R$ can be obtained from $L$. Now, $R$ is a well-constrained problem which can be solved recursively.

\section{(c) Merge $L$ and $R$ to obtain $N$.}

In what below, we will give a detailed analysis of the merging process for bi-connected and tri-connected graphs. The general case can be reduced to one of these cases. The reason is that the relative position of two rigid bodies can be fixed by merging them along a minimal rigid body shared by them, and a minimal rigid body contains two or three primitives [2].

\subsection{Merge bi-connected constraint graphs}

Let the separating pair be $\{a, b\}$ in a bi-connected graph $G$. The split subgraphs of $G$ are two rigid bodies $R_{1}$ and $R_{2}$. Now we show how to assemble $R_{1}$ and $R_{2}$.

The problem can be classified into the following six cases according to the types of $a$ and $b$.

1. The vertices $a$ and $b$ are two points. The relative position of $R_{1}$ and $R_{2}$ can not be fixed because $R_{1}$ may rotate around the segment $a b$ assuming that $R_{2}$ is fixed. Thus $R_{1} \cup R_{2}$ is not a rigid body anymore although structurally well-constrained

2. The vertices $a$ and $b$ are two planes. There is at least one translation degree of freedom between $R_{1}$ and $R_{2}$. Thus $R_{1} \bigcup R_{2}$ is not a rigid body.

3. The vertices $a$ and $b$ are a point and a plane. The relative position of $R_{1}$ and $R_{2}$ can not be fixed because there is a rotation degree of freedom left. Thus $R_{1} \cup R_{2}$ is not a rigid body anymore.

4. The vertices $a$ and $b$ are two lines. If $a$ and $b$ are parallel, the relative position of $R_{1}$ and $R_{2}$ can not be fixed. If $a$ and $b$ are not parallel, the relative position of $R_{1}$ and $R_{2}$ can be fixed.

5. The vertices $a$ and $b$ are a point and a line. If the distance between $a$ and $b$ is zero, the relative position of $R_{1}$ and $R_{2}$ can not be fixed. If the distance between $a$ and $b$ is not zero, the relative position of $R_{1}$ and $R_{2}$ can be fixed.

6. The vertices $a$ and $b$ are a plane and a line. When $a$ and $b$ are parallel, the relative position of $R_{1}$ and $R_{2}$ can not be fixed. If $a$ and $b$ are perpendicular to each other, the relative position of $R_{1}$ and $R_{2}$ can not be fixed. Let the angle between $a$ and $b$ be $\angle(a, b)$, if $0<|\cos (\angle(a, b))|<1$, the relative position of $R_{1}$ and $R_{2}$ can be fixed.

THEOREM 5.1. Let $\{a, b\}$ be the separating pair in a biconnected graph $G$ in $3 D, R_{1}$ and $R_{2}$ the split subgraphs of $G$ which are two rigid bodies. Then $R$ is a rigid body if and only if $\{a, b\}$ are of the following three cases: a point and $a$ line which are not incident; a plane and a line which are not parallel, perpendicular or incident to each other; two lines which are not parallel to each other.

The problem in Fig. 3(a) is the double banana problem, where the weight of each vertex is 3 and the weight of each edge is $1 .\left\{p_{1}, p_{5}\right\}$ is a separating pair. $G_{1}$ induced by $\left\{p_{1}, p_{2}, p_{3}, p_{4}, p_{5}\right\}$ and $G_{2}$ induced by $\left\{p_{1}, p_{5}, p_{6}, p_{7}, p_{8}\right\}$ are structurally well-constrained. But the cut graph induced by $\left\{p_{1}, p_{5}\right\}$ is not structurally well-constrained. According to Theorem 5.1, $G$ is not a rigid body if $G_{1}$ and $G_{2}$ are rigid bodies. For a recent work to characterize such illconditioned problems, please consult [12]. 


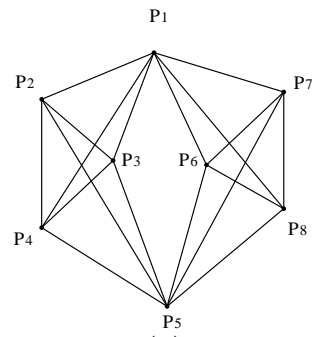

(a)

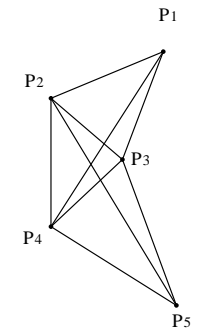

(b)

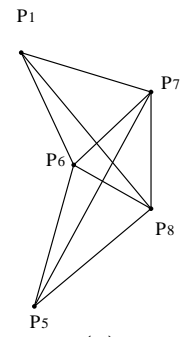

(c)
Figure 3: Double banana problem

\subsection{Merge tri-connected constraint graphs}

Let $V_{s}=\{a, b, c\}$ be the separating triplet of $G=(V, E, \omega)$, $G_{s}=\left(V_{s}, E_{s}, \omega\right)$ the subgraph induced by $V_{s}, G_{1}$ and $G_{2}$ the split graphs which are rigid bodies. We will try to assemble $R_{1}$ and $R_{2}$. The problem can be classified into the following ten cases according to the types of $a, b$ and $c$.

1. The vertices $a, b$ and $c$ are three non-collinear points. The relative position of $R_{1}$ and $R_{2}$ can be fixed and $R_{1} \cup R_{2}$ is a rigid body.

2. $a, b$ and $c$ are three planes. If two of them are parallel, the relative position of $R_{1}$ and $R_{2}$ can not be fixed. If the three planes intersect with each other and the three intersection lines are parallel, the relative position of $R_{1}$ and $R_{2}$ can not be fixed. Otherwise the relative position of $R_{1}$ and $R_{2}$ can be fixed.

3. The vertices $a, b$ and $c$ are three lines. If the three lines are parallel to each other, the relative position of $R_{1}$ and $R_{2}$ can not be fixed. Otherwise the relative position of $R_{1}$ and $R_{2}$ can be fixed.

4. The vertices $a$ and $b$ are two points and $c$ is a plane. The relative position of $R_{1}$ and $R_{2}$ can be fixed.

5. The vertices $a$ and $b$ are two points and $c$ is a line. If both the two points are on the line, the relative position of $R_{1}$ and $R_{2}$ can not be fixed. Otherwise the relative position of $R_{1}$ and $R_{2}$ can be fixed.

6. The vertex $a$ is a point, $b$ and $c$ are two planes. The relative position of $R_{1}$ and $R_{2}$ can be fixed.

7. The vertex $a$ is a point, $b$ and $c$ are two lines. The relative position of $R_{1}$ and $R_{2}$ can be fixed.

8. $a$ and $b$ are two planes and $c$ is a line. If $a \| b$ and the line is perpendicular to the planes, the relative position of $R_{1}$ and $R_{2}$ can not be fixed. If the line is parallel to the two planes, the relative position of $R_{1}$ and $R_{2}$ can not be fixed. Otherwise the relative position of $R_{1}$ and $R_{2}$ can be fixed.

9. The vertices $a$ and $b$ are two lines and $c$ is a plane. If the two lines are parallel and on the plane, the relative position of $R_{1}$ and $R_{2}$ can not be fixed. Otherwise the relative position of $R_{1}$ and $R_{2}$ can be fixed.

10. Vertex $a$ is a point, $b$ is a plane, and $c$ is a line. If $a$ is on $c$ and $c$ is perpendicular to $b$, the relative position of $R_{1}$ and $R_{2}$ can not be fixed. Otherwise, the relative position of $R_{1}$ and $R_{2}$ can be fixed.
For $k$-connected graph $(k>3)$, we can always select three geometric primitives in the separating $k$-tuple to merge the two rigid bodies with the three primitives.

\section{REFERENCES}

[1] I. Fudos and C.M. Hoffmann, A Graph-constructive Approach to Solving Systems of Geometric Constraints, ACM Transactions on Graphics, 16(2), 179-216, 1997.

[2] X.S. Gao and G.F. Zhang, Geometric Constraint Solving via C-tree Decomposition, Proc.ACM SM03, 45-55, ACM Press, New York, 2003.

[3] J.E. Hopcroft and R.E. Tarjan, Dividing a Graph into Triconnected Components, SIAM J. Comput. 135-158, 1973.

[4] C.M. Hoffmann, A. Lomonosov and M. Sitharam, Decomposition Plans for Geometric Constraint Systems, II: New Algorithms, 31, 409-427, J. of Symbolic Computation, 2001.

[5] R. Joan-Arinyo, A. Soto-Riera, S. Vila-Marta and J. Vilaplan-Pastó, Revisiting Decomposition Analysis of Geometric Constraint Graphs, Proc. ACM SM02, 105-115, ACM Press, New York, 2002.

[6] D. Jungnickel, Graphs, Graphen, Netzwerke, und Algorithmen, Springer, Berlin, 1999.

[7] A. Kanevsky and V. Ramachandran, Improved Algorothms for Graph Four-connectivity, Proc. 28th Ann. IEEE Symp. Foundations of Computer Science, Los Angeles, 252-259, 1987.

[8] H. Lamure and D. Michelucci, Qualitative Study of Geometric Constraints, in Geometric Constraint Solving and Applications, 234-258, Springer, Berlin, 1998.

[9] R.S. Latham and A.E. Middleditch, Connectivity Analysis: a Tool for Processing Geometric Constraints, Computer-Aided Design, 28(11), 917-928, 1994.

[10] J. Van Leeuwen, Handbook of Theoretical Computer Science(Volume A): Algorithms and Complexity, Elsevier Science Publishers B.V. 1990.

[11] J. Owen, Algebraic Solution for Geometry from Dimensional Constraints, in ACM Symp., Found of Solid Modeling, ACM Press, New York, 397-407, 1991.

[12] M. Sitharam and Y. Zhou, A Tractable, Approximate Characterization of Combinatorial Rigidity in 3D, Automated Deduction in Geometry, ADG 2004, September, 2004Florida University.

[13] G.F. Zhang and X.S. Gao, Geometric Constraint Solving Based on Connectivity of Graph, Computer-Aided Design and Applications, 1(1-4), 469-476. 